\title{
Sample Functions of Stochastic Processes with Stationary Independent Increments
}

\author{
R. M. BLUMENTHAL \& R. K. GETOOR ${ }^{1}$
}

\author{
Communicated by W. FeLLeR
}

1. Introduction. Let $X=\{X(t) ; t \geqq 0\}$ be a stochastic process in $R_{N}$ with stationary independent increments. We are interested in problems of the following type concerning the sample functions of $X$ : (1) local HöLDER conditions; (2) conditions under which the $\gamma$-variation of $X$ over [0,1] is finite (or infinite); (3) the relationship between $\operatorname{dim} E$ and $\operatorname{dim} X(E)$ when $E$ is a BorEL subset of $[0,1]$. (Here $\operatorname{dim} A$ stands for the HAusd onFF-Besicovitch dimension of the set $A$.) In case $X$ is a stable process of index $\alpha$, then the behavior of the sample functions relative to these three properties can be described to a large extent in terms of the single parameter $\alpha$. See, for example, [1], [2], and [8]. In the present paper we define and investigate some parameters of this type for the general class of processes considered here.

We first normalize $X$ in a natural way and then define two indices $\beta$ (section 2 ) and $\beta^{\prime}$ (section 5) in such a way that if $X$ is stable of index $\alpha$ then $\beta^{\prime}=\beta=\alpha$. In section 3 we prove that

$$
\lim _{t \rightarrow 0} t^{-1 / \alpha} X(t)=0 \quad \text { if } \quad \alpha>\beta
$$

and

$$
\limsup _{t \rightarrow 0} t^{-1 / \alpha}|X(t)|=\infty \quad \text { if } \quad \alpha<\beta,
$$

both statements holding with probability one. In section 4 we show that the $\gamma$-variation of $X$ over $[0,1]$ is infinite with probability one if $\gamma<\beta$ and is finite with probability one if $\beta<\gamma \leqq 1$. Section 5 contains further information on local HöLder conditions. The subordinators (a special class of processes of the type under consideration) are studied in section 6 . Somewhat more precise results are obtained in this special case. Section 7 is devoted to the construction of an example. Finally we prove in section 8 that if $\operatorname{dim} E=\alpha$, then, except

\footnotetext{
1 The first author was supported by the Office of Naval Research. The second is a National Science Foundation Postdoctoral Fellow.
} 
for a slight modification if $N=1$ and $\beta^{\prime}>1, P\left[\operatorname{dim} X(E) \geqq \min \left(\alpha \beta^{\prime}, N\right)\right]=1$, and if $\beta<1$ then $P[\operatorname{dim} X(E) \leqq \alpha \beta]=1$.

We would like to thank Professor H. P. McKean for several valuable comments and suggestions.

2. Preliminaries. Let $X=\{X(t) ; t \geqq 0\}$ be a stochastic process in $R_{N}$ with stationary independent increments. If $\phi_{t-s}$ is the $N$-dimensional characteristic function of $X(t)-X(s)$ with $t>s$ then $\phi_{t-s}(y)=\exp [-(t-s) \psi(y)]$ where

$$
\psi(y)=i(a, y)+\frac{1}{2} y S y^{\prime}+\int\left[1-e^{i(x, y)}+\frac{i(x, y)}{1+|x|^{2}}\right] \nu(d x),
$$

with $a$ in $R_{N}, S$ a non-negative definite symmetric $N \times N$ matrix, and $\nu$ a (BoreL) measure on $R_{N}$ satisfying

$$
\int \frac{|x|^{2}}{1+|x|^{2}} \nu(d x)<\infty
$$

The quantities $a, S$, and $\nu$ appearing in (2.1) are uniquely determined by $\phi$, and hence by the process $X$. Here and throughout this paper, integrals are taken over all of $R_{N}$ unless explicitly stated otherwise. The notation $(x, y)$ denotes the usual inner product in $R_{N}$ and $|x|=(x, x)^{1 / 2}$ is the usual Euclidean norm. Formula (2.1) is well-known; see Bochner [3] and Takano [10]. We will assume henceforth that our process has no Gaussian part, that is $S=0$.

The function $\psi$ is called the exponent of $X$ and $\nu$ is called the Lèvy measure of $X$. We will assume throughout that $X(0)=0$.

It is a standard fact that we may assume the sample functions of $X$ to have certain regularity properties. To be precise we assume that almost all sample functions have left and right hand limits everywhere and are right continuous for all $t \geqq 0$. Our process is defined over some basic probability space $(\Omega, \mathfrak{F}, P)$. Usually we will suppress the $\omega$ 's in our notation.

We now define the index, $\beta$, of the process $X$.

Definition 2.1.

$$
\beta=\inf \left\{\alpha>0: \int_{|x|<1}|x|^{\alpha} \nu(d x)<\infty\right\} .
$$

Clearly $0 \leqq \beta \leqq 2$ and it is easy to see that if $X$ is a stable process in $R_{N}$ of index $\alpha$ then $\beta=\alpha$. (The Lìvy measure of a stable process of index $\alpha$ has the form

$$
\nu(d x)=\frac{d r}{r^{1+\alpha}} \mu(d \theta)
$$

where $x=(r, \theta)$ with $r \geqq 0$ and $\theta$ a point on the surface, $S_{N}$, of the unit sphere in $R_{N}$. Here $\mu$ is an arbitrary finite (BorEL) measure on $S_{N}$.) 
We will further normalize our process as follows: if $\beta<1$, or even if $\int_{|x|<1}|x| \nu(d x)<\infty$, then by removing a linear term from $X$ we may write the exponent $\psi$ in the form

$$
\psi(y)=\int\left[1-e^{i(x, y)}\right] \nu(d x) .
$$

We will always assume that $\psi$ has the form (2.3) if $\int_{|x|<1}|x| \nu(d x)<\infty$; if this integral is infinite then $\psi$ has the form (2.1) with $S=0$. The removal of the linear term from $X$ in these cases is quite natural since otherwise the behavior of $X(t)$ near $t=0$ is dominated by the behavior of the linear term.

The following characterization of $\beta$ will be useful later on.

Theorem 2.1. Let $G(r)=\nu\{x:|x|>r\}$; then

$$
\beta=\inf \left\{\alpha>0: r^{\alpha} G(r) \rightarrow 0 \text { as } r \rightarrow 0\right\} .
$$

Proof. Denote the infimum in Theorem 2.1 by $\gamma$. Suppose $\alpha>\gamma$ and choose $\alpha^{\prime}$ so that $\alpha>\alpha^{\prime}>\gamma$. Then $r^{\alpha^{\prime}} G(r) \rightarrow 0$. But

$$
\int_{\epsilon<|x| \leqq 1}|x|^{\alpha} \nu(d x)=-\int_{\epsilon}^{1} r^{\alpha} d G(r)=\epsilon^{\alpha} G(\epsilon)-G(1)+\alpha \int_{\epsilon}^{1} r^{\alpha-\alpha^{\prime}-1} r^{\alpha^{\prime}} G(r) d r,
$$

so as $\epsilon \rightarrow 0$ the right side approaches a finite limit. Therefore $\alpha \geqq \beta$ and hence $\beta \leqq \gamma$. If $\alpha>\beta$ then

$$
-\int_{0}^{\delta} r^{\alpha} d G(r) \geqq-\int_{\epsilon}^{\delta} r^{\alpha} d G(r) \geqq \epsilon^{\alpha}[G(\epsilon)-G(\delta)]
$$

Hence

$$
\limsup _{\epsilon \rightarrow 0} \epsilon^{\alpha} G(\epsilon) \leqq-\int_{0}^{\delta} r^{\alpha} d G(r)
$$

and since $\delta>0$ is arbitrary it follows that $r^{\alpha} G(r) \rightarrow 0$ as $r \rightarrow 0$. Thus $\alpha \geqq \gamma$ and hence $\beta \geqq \gamma$. This concludes the proof of Theorem 2.1.

3. Hölder Conditions. In this section we obtain several alternate forms for the index $\beta$ and obtain information on the behavior of $X(t)$ near $t=0$ in terms of the index.

Lemma 3.1. If $\alpha>\beta$, then $|y|^{-\alpha}|\psi(y)| \rightarrow 0$ as $|y| \rightarrow \infty$.

Proof. It is known that $|y|^{-2}|\psi(y)| \rightarrow 0$ as $|y| \rightarrow \infty$ in any case (Theorem 3.4.2 of [3]). Thus we may assume $\beta<2$. Moreover $|\psi(y)| \leqq|\operatorname{Re} \psi(y)|+|\operatorname{Im} \psi(y)|$. If $\alpha>\beta$ choose $\alpha^{\prime}<2$ such that $\alpha>\alpha^{\prime}>\beta$; then using the elementary inequality $1-\cos t \leqq 2 t^{\alpha^{\prime}}$ we obtain $|y|^{-\alpha}|\operatorname{Re} \psi(y)| \rightarrow 0$.

If $\beta<1$ and $\alpha>\beta$ choose $\alpha^{\prime}<1$ such that $\alpha>\alpha^{\prime}>\beta$; then the inequality $|\sin t| \leqq t^{\alpha^{\prime}}$ implies that

$$
|\operatorname{Im} \psi(y)| \leqq|y|^{\alpha^{\prime}} \int_{|x| \leqq 1}|x|^{\alpha^{\prime}} \nu(d x)+\int_{|x|>1} \nu(d x) .
$$


Hence $|y|^{-\alpha}|\operatorname{Im} \psi(y)| \rightarrow 0$. Finally if $\beta \geqq 1, \alpha^{\prime}<2$ and $\beta<\alpha^{\prime}<\alpha$, the inequality $|t-\sin t| \leqq 2 t^{\alpha^{\prime}}$ implies that

$$
|\operatorname{Im} \psi(y)| \leqq K|y|+2|y|^{\alpha^{\prime}} \int_{|x|<1}|x|^{\alpha^{\prime}} \nu(d x)+M,
$$

where $K$ and $M$ are constants. Thus the desired result follows again in this case.

Let $F(t, A)$ be the distribution of the random variable $X(t)$, that is,

$$
e^{-t \psi(x)}=\int e^{i(x, y)} F(t, d y) .
$$

Also let $f_{\alpha}(x)$ be the continuous density of an $N$-dimensional random variable having a symmetric stable distribution of index $\alpha$ where $0<\alpha \leqq 2$, that is

$$
e^{-|x|^{\alpha}}=\int e^{i(x, y)} f_{\alpha}(y) d y
$$

Lemma 3.2. If $\beta<\alpha<2$ and if

$$
A(t)=\int e^{-|x|^{\alpha}} F(t, d x)
$$

then $t^{-1}[1-A(t)] \rightarrow k<\infty$ as $t \rightarrow 0$.

Proof. We write

$$
\begin{aligned}
A(t) & =\int F(t, d x) \int e^{i(y, x)} f_{\alpha}(y) d y \\
& =\int e^{-t \psi(y)} f_{\alpha}(y) d y
\end{aligned}
$$

and therefore

$$
t^{-1}[1-A(t)]=\int t^{-1}\left[1-e^{-t \psi(y)}\right] f_{\alpha}(y) d y .
$$

Since $\operatorname{Re} \psi(y) \geqq 0$ it is an elementary fact that $t^{-1}\left|1-e^{-t \psi(y)}\right| \leqq|\psi(y)|$ for all $y$. We now show that $\int|\psi(y)| f_{\alpha}(y) d y<\infty$. Since $|\psi|$ is a continuous function, the convergence of this integral depends on what happens for $|y|$ large. We have shown ([1], Theorem 2.1) that $f_{\alpha}(y) \sim C_{\alpha}|y|^{-N-\alpha}$ as $|y| \rightarrow \infty$ if $\alpha<2$. Also if $\beta<\alpha^{\prime}<\alpha$, an application of Lemma 3.1 shows that $|\psi(y)| \leqq|y|^{\alpha^{\prime}}$ for $|y|$ sufficiently large. Thus for large $R$ we have

$$
\int_{|y| \geqq R}|\psi(y)| f_{\alpha}(y) d y \leqq K \int_{R}^{\infty} r^{\alpha^{\prime}} r^{-N-\alpha} r^{N-1} d r<\infty .
$$

Combining these observations with the bounded convergence theorem we see that

$$
t^{-1}[1-A(t)] \rightarrow \int \psi(y) f_{\alpha}(y) d y=k
$$


as $t \rightarrow 0$, and that $|k|<\infty$. Since $t^{-1}[1-A(t)] \geqq 0$ for all $t>0$ it follows that $k$ is real and non-negative. This completes the proof of Lemma 3.2.

The main result of this section is that for any $\alpha>\beta, X(t)$ satisfies a HöLDER condition of order $\alpha^{-1}$ at $t=0$. More precisely we have the following theorem.

Theorem 3.1. If $\alpha>\beta$ then $t^{-1 / \alpha} X(t) \rightarrow 0$ as $t \rightarrow 0$ with probability one.

Proof. In this proof we will use two results of KHIntchine [7]. The first is that if $\{Y(t) ; t \geqq 0\}$ is a process in $R_{1}$ with stationary independent increments, if $u(t)$ is a positive non-decreasing function on $0<t<1$, and if $P_{c}(t)=$ $P[|Y(t)|>c u(t)]$ then $Y(t) / u(t) \rightarrow 0$ with probability one as $t \rightarrow 0$ if and only if $\int_{0}^{1} t^{-1} P_{c}(t) d t<\infty$ for all $c>0$. The second result is that if $Y$ has no Gaussian part and $u(t)=\left(t \log \log t^{-1}\right)^{1 / 2}$ then $Y(t) / u(t) \rightarrow 0$ with probability one as $t \rightarrow 0$. These results are easily extended to processes in $R_{N}$.

Suppose first that $\beta<2$. It clearly suffices to prove the result for $\alpha$ satisfying $\beta<\alpha<2$ in this case. Given $\eta>0$ we have, using the notation of Lemma 3.2,

$$
\begin{aligned}
1-A(t) & =\int\left(1-e^{-|x|^{\alpha}}\right) F(t, d x) \\
& \geqq \int_{|x|>\eta^{1 / \alpha}}\left(1-e^{-|x|^{\alpha}}\right) F(t, d x) \\
& \geqq\left(1-e^{-\eta}\right) P\left[|X(t)|>\eta^{1 / \alpha}\right] .
\end{aligned}
$$

But $\eta^{-1}\left(1-e^{-\eta}\right) \rightarrow 1$ as $\eta \rightarrow 0$ and $t^{-1}[1-A(t)] \rightarrow k$ as $t \rightarrow 0$, and therefore if $t$ and $\eta$ are sufficiently small we have

$$
P\left[|X(t)|^{\alpha}>\eta\right] \leqq K_{\alpha} t \eta^{-1}
$$

where $K_{\alpha}$ is a positive constant.

Let $u(t)=t^{1 / \alpha}$ and choose $\alpha^{\prime}$ such that $\beta<\alpha^{\prime}<\alpha$; then for any $c>0$ we have

$$
\begin{aligned}
P_{c}(t) & =P\left[|X(t)|>c t^{1 / \alpha}\right] \\
& =P\left[|X(t)|^{\alpha^{\prime}}>c^{\alpha^{\prime}} t^{\alpha^{\prime / \alpha}}\right] \\
& \leqq K_{\alpha^{\prime}} c^{-\alpha^{\prime}} t^{1-\alpha^{\prime} / \alpha}
\end{aligned}
$$

provided $t$ is sufficiently small (how small depending on $c$ ). Hence

$$
\int_{0}^{1} t^{-1} P_{c}(t) d t<\infty
$$

and therefore $t^{-1 / \alpha} X(t) \rightarrow 0$ with probability one, by the first result of KHINTChINe.

The result in the case $\beta=2$ follows from KHINTchine's second result and the fact that $\left(t \log \log t^{-1}\right)^{1 / 2} \leqq t^{1 / \alpha}$ for all $\alpha>2$ if $t$ is sufficiently small. This completes the proof of Theorem 3.1. 
We will now derive an expression for $\beta$ directly in terms of $\psi$. We begin with the following lemma.

Lemma 3.3. The integrals $\int \operatorname{Re} \psi(y) f_{\alpha}(y) d y \leqq \int|\psi(y)| f_{\alpha}(y) d y$ converge if $\beta<\alpha \leqq 2$ (or $\alpha=2$ if $\beta=2$ ) and diverge if $0<\alpha<\beta$.

Proof. We have already seen, (3.4), in the proof of Lemma 3.2 that $\int|\psi(y)| f_{\alpha}(y) d y<\infty$ if $\alpha>\beta$ and $\alpha<2$. Since $f_{2}(y)=A e^{-B|y| z}$ where $A$ and $B$ are positive constants and $|\psi(y)|=o(|y|)^{2}$ as $|y| \rightarrow \infty$, the assertions concerning convergence are established. On the other hand

$$
\begin{aligned}
\int \operatorname{Re} \psi(y) f_{\alpha}(y) d y & =\int f_{\alpha}(y) d y \int[1-\cos (x, y)] \nu(d x) \\
& =\int\left[1-e^{-|x| \alpha}\right] \nu(d x),
\end{aligned}
$$

and this last integral certainly diverges if $\alpha<\beta$.

Theorem 3.2. The index

$$
\begin{aligned}
\beta=\inf \left\{\alpha \geqq 0:|y|^{-\alpha}|\psi(y)|\right. & \rightarrow 0 \text { as }|y| \rightarrow \infty\} \\
& =\inf \left\{\alpha \geqq 0:|y|^{-\alpha} \operatorname{Re} \psi(y) \rightarrow 0 \text { as }|y| \rightarrow \infty\right\} .
\end{aligned}
$$

Proof. According to Lemma 3.1 if $\alpha>\beta$ then

$$
|y|^{-\alpha} \operatorname{Re} \psi(y) \leqq|y|^{-\alpha}|\psi(y)| \rightarrow 0
$$

as $|y| \rightarrow \infty$. Conversely suppose $0 \leqq \alpha<\beta$; then choosing $\alpha^{\prime}$ such that $\alpha<\alpha^{\prime}<\beta$, Lemma 3.3 and the fact that $\operatorname{Re} \psi$ is continuous imply that

$$
\int_{|x|>R} \operatorname{Re} \psi(y) f_{\alpha^{\prime}}(y) d y=\infty
$$

for all $R>0$. But if $|y|^{-\alpha} \operatorname{Re} \psi(y)$ is bounded near $|y|=\infty$ this leads to a contradiction since $f_{\alpha^{\prime}}(y) \sim c|y|^{-N-\alpha^{\prime}}$. Thus if $\alpha<\beta$ there exists a sequence $\left\{y_{n}\right\}$ such that $\left|y_{n}\right| \rightarrow \infty$ and $\left|y_{n}\right|^{-\alpha} \operatorname{Re} \psi\left(y_{n}\right) \rightarrow \infty$. This proves Theorem 3.2.

The following theorem complements the result of Theorem 3.1.

Theorem 3.3. If $\alpha<\beta$ then

$$
\limsup _{t \rightarrow 0} t^{-1 / \alpha}|X(t)|=\infty
$$

with probability one.

Proof. Choose $\gamma$ such that $\alpha<\gamma<\beta$. If $\rho_{t}(y)$ is the characteristic function of $t^{-1 / \gamma} X(t)$, then $\rho_{t}(y)=\exp \left[-t \psi\left(t^{-1 / \gamma} y\right)\right]$. According to Theorem 3.2 there exists a sequence $\left\{y_{n}\right\}$ with $\left|y_{n}\right| \rightarrow \infty$ such that $\left|y_{n}\right|^{-\gamma} \operatorname{Re} \psi\left(y_{n}\right) \rightarrow \infty$. Set $t_{n}=$ $\left|y_{n}\right|^{-\gamma}$ and $\theta_{n}=\left|y_{n}\right|^{-1} y_{n}$. Then $t_{n} \rightarrow 0, \theta_{n}$ is in $S_{N}$, the unit sphere in $R_{N}$, and $\rho_{t_{n}}\left(\theta_{n}\right) \rightarrow 0$. Consider the sequence $\left\{t_{n}^{-1 / \alpha}\left|X\left(t_{n}\right)\right|\right\}$. Since thelim sup of this sequence 
depends only on the process for arbitrarily small values of $t$, it follows from the zero-one law (HuNT [6]) that it is a constant, say $c$, with probability one. If $c=\infty$ we are finished. But if $c<\infty$ then $t_{n}^{-1 / \gamma}\left|X\left(t_{n}\right)\right| \rightarrow 0$ with probability one. Thus the sequence of characteristic functions $\rho_{t_{n}}$, must approach one uniformly on any bounded subset of $R_{N}$. But this contradicts the fact that $\rho_{t_{n}}\left(\theta_{n}\right) \rightarrow 0$. Therefore $c=\infty$ and Theorem 3.3 is established.

4. The $\gamma$-variation of $X$. Let $f$ be a function defined on $-\infty<a \leqq$ $l \leqq b<+\infty$ taking values in $R_{N}$. For each $\gamma>0$ we define

$$
V_{\gamma}(f ; a, b)=\sup \sum_{j=1}^{m}\left|f\left(t_{j}\right)-f\left(t_{j-1}\right)\right|^{\gamma}
$$

where the supremum is taken over all finite subdivisions $a=t_{0}<t_{1}<\cdots$ $<t_{m}=b$. The (possibly infinite) number $V_{\gamma}(f ; a, b)$ is called the $\gamma$-variation of $f$ over $[a, b]$. Clearly if $V_{\gamma}(f ; a, b)<\infty$ then $V_{\gamma},(f ; a, b)<\infty$ for all $\gamma^{\prime}>\gamma$.

If $X$ is a process of the type we are considering, it is not difficult to see that $V_{\gamma}(X(\cdot, \omega) ; a, b)$ is a measurable function of $\omega$ and hence a random variable; see ([1], Lemma 2.2). We are going to investigate the random variable $V_{\gamma}(X)=$ $V_{\gamma}(X(\cdot, \omega) ; 0,1)$. Our method is that of Bochner [3, Sec. 5.3]. For the sake of clarity we will reproduce some of his arguments instead of giving detailed references to the relevant parts of his development.

Theorem 4.1. If $\gamma<\beta$ then $V_{\gamma}(X)=\infty$ with probability one.

Proof. For each positive integer $n$ let

$$
F_{n}(\omega)=\sum_{j=1}^{2^{n}}\left|X\left(j 2^{-n}, \omega\right)-X\left((j-1) 2^{-n}, \omega\right)\right|^{\gamma},
$$

and set $F(\omega)=\sup F_{n}(\omega)$. Certainly $F(\omega) \leqq V_{\gamma}(X)$ and so it is sufficient to show that $F(\omega)=\infty$ for almost all $\omega$. Set

$$
A(t)=e^{-B(t)}=\int e^{-|x| \gamma} F(t, d x) \leqq \int e^{-t \operatorname{Re} \psi(x)} f_{\gamma}(x) d x ;
$$

then

$$
t^{-1}[1-A(t)] \geqq \int t^{-1}\left[1-e^{-t \operatorname{Re} \psi(x)}\right] f_{\gamma}(x) d x \rightarrow \int \operatorname{Re} \psi(x) f_{\gamma}(x) d x
$$

as $t \rightarrow 0$. Since $\gamma<\beta$, Lemma 3.3 implies that this last integral diverges and hence $t^{-1}[1-A(t)] \rightarrow \infty$ as $t \rightarrow 0$. Therefore $t^{-1} B(t) \rightarrow \infty$ as $t \rightarrow 0$. Using the fact that $X$ has stationary independent increments we obtain

$$
\mathcal{E}\left\{e^{-F}\right\} \leqq \mathcal{E}\left\{e^{-F_{n}}\right\}=\left[A\left(2^{-n}\right)\right]^{2^{n}}=e^{-2^{n} B\left(2^{-n}\right)} \rightarrow 0
$$

as $n \rightarrow \infty$. Thus $F=\infty$ with probability one.

Theorem 4.2. If $\beta<\gamma \leqq 1$ then $V_{\gamma}(X)$ is finite with probability one. 
Proof. Using the same notation as in the proof of Theorem 4.1, we have, since $\gamma \leqq 1$, that $V_{\gamma}(X)=F=\lim _{n \rightarrow \infty} F_{n}$. Define for $t>0$ and $u>0$

$$
\begin{aligned}
A(t, u) & =e^{B(t, u)}=\int e^{-u|x| \gamma} F(t, d x) \\
& =\int e^{-t \psi(x)} f_{\gamma}\left(u^{-1 / \gamma} x\right) u^{-N / \gamma} d x ;
\end{aligned}
$$

then since $\gamma>\beta$ we have, as in the proof of Lemma 3.2,

$$
t^{-1}[1-A(t, u)] \rightarrow \int \psi\left(u^{1 / \gamma} x\right) f_{\gamma}(x) d x
$$

as $t \rightarrow 0$. If we choose $\alpha$ so that $\beta<\alpha<\gamma$ then Theorem 3.2 implies that

$$
\left|\psi\left(u^{1 / \gamma} x\right)\right| \leqq M\left(1+|x|^{\alpha}\right)
$$

for $u \leqq 1$ and $\psi\left(u^{1 / \gamma} x\right) \rightarrow 0$ as $u \rightarrow 0$. Therefore, by the bounded convergence theorem,

$$
\lim _{u \rightarrow 0} \lim _{t \rightarrow 0} t^{-1} B(t, u)=\lim _{u \rightarrow 0} \lim _{t \rightarrow 0} t^{-1}[1-A(t, u)]=0
$$

Now

$$
\lim _{u \rightarrow 0} \varepsilon e^{-u F}=\lim _{u \rightarrow 0} \lim _{n \rightarrow \infty} \varepsilon e^{-u F_{n}}=\lim _{u \rightarrow 0} \lim _{n \rightarrow \infty} e^{-2^{n} B\left(2^{-n}, u\right)}=1 .
$$

But $\lim _{u \rightarrow 0} \varepsilon^{-u F}=P[F<\infty]$ and thus $V_{\gamma}(X)$ is finite with probability one.

It is reasonable to conjecture that $V_{\gamma}(X)$ is finite with probability one provided $\gamma>\beta$ without the restriction $\beta<1$. However we don't even know this to be the case for the general stable processes. We established this result for the symmetric stable processes in [1], Theorem 4.1 .

5. The lower indices. In view of theorem 3.2 it is natural to consider the number

$$
\beta^{\prime \prime}=\sup \left\{\alpha \geqq 0:|y|^{-\alpha} \operatorname{Re} \psi(y) \rightarrow \infty \quad \text { as } \quad|y| \rightarrow \infty\right\} .
$$

Actually it will turn out that the number

$$
\beta^{\prime}=\sup \left\{\alpha \geqq 0: \int|x|^{\alpha-N} \frac{1-e^{-R \Theta \psi(x)}}{\operatorname{Re} \psi(x)} d x<\infty\right\}
$$

is more relevant to the considerations which follow, but in any event we call $\beta^{\prime}$ and $\beta^{\prime \prime}$ the lower indices and we have the following relations among the various indices.

Theorem 5.1. $0 \leqq \beta^{\prime \prime} \leqq \beta^{\prime} \leqq \beta$. Moreover, for any $R>0$,

$$
\beta^{\prime} \geqq \sup \left\{\alpha \geqq 0: \int_{|x|>R}|x|^{\alpha-N}[\operatorname{Re} \psi(x)]^{-1} d x<\infty\right\} \text {. }
$$


Proof. The second statement is immediate since $\operatorname{Re} \psi \geqq 0$ and

$$
\int_{|x| \leqq R}|x|^{\alpha-N} \frac{1-e^{-\operatorname{Re} \psi(x)}}{\operatorname{Re} \psi(x)} d x<\infty
$$

for any $R>0$ and $\alpha>0$. Also if $\alpha<\beta^{\prime \prime}$, then choosing $\alpha^{\prime}$ such that $\alpha<\alpha^{\prime}<\beta^{\prime \prime}$, we have $\operatorname{Re} \psi(x) \geqq|y|^{\alpha^{\prime}}$ for $|y|$ large. Combining this with the second statement of the theorem yields $\alpha \leqq \beta^{\prime}$ and therefore $\beta^{\prime \prime} \leqq \beta^{\prime}$. Finally if $\alpha>\beta$ we know from Theorem $3.2 \operatorname{Re} \psi(x) \leqq|x|^{\alpha}$ for $|x|$ sufficiently large. This easily implies that

$$
\int|x|^{\alpha-N} \frac{1-e^{-\operatorname{Re} \psi(x)}}{\operatorname{Re} \psi(x)} d x=\infty,
$$

and hence $\alpha \geqq \beta^{\prime}$. Therefore $\beta^{\prime} \leqq \beta$.

We will show in section 7 that $\beta^{\prime}<\beta$ is possible, and later on in this section that $\beta^{\prime \prime}<\beta^{\prime}$ is also possible. It is easy to see that for a stable process of index $\alpha$ we have $\beta^{\prime \prime}=\beta^{\prime}=\beta=\alpha$ Also Theorems 3.2 and 5.1 imply that if there exists a constant $\alpha$ such that $|y|^{-\alpha+\epsilon} \operatorname{Re} \psi(y) \rightarrow \infty$ and $|y|^{-\alpha-\epsilon} \operatorname{Re} \psi(y) \rightarrow 0$ as $|y| \rightarrow \infty$ for all $\epsilon>0$, then $\beta^{\prime \prime}=\beta^{\prime}=\beta=\alpha$.

Theorem 5.2. If $\alpha<\beta^{\prime}$ and $\alpha<N$, then $\int_{0}^{1} \mathcal{E}\left\{|X(t)|^{-\alpha}\right\} d t<\infty$. Moreover, if $\psi$ is real valued, then this integral is infinite if $\alpha>\beta^{\prime}$.

Proof. First of all, let us note that if $F$ is any $N$-dimensional distribution function and $\phi$ is its characteristic function, then for each $\alpha>0$

$$
2^{\alpha / 2-1} \Gamma\left(\frac{\alpha}{2}\right) \int|y|^{-\alpha} F(d y)=(2 \pi)^{-N / 2} \int_{0}^{\infty} u^{\alpha-1} d u \int e^{-\frac{1}{2}|y|^{2}} \phi(u y) d y .
$$

This equality is obtained by replacing $\phi$ in the right side of (5.3) by its expression as a FouRIER integral and then performing a routine calculation. Thus, if $\alpha<N$, we have

$$
J(\alpha)=\int_{0}^{1} \varepsilon\left\{|X(t)|^{-\alpha}\right\} d t \leqq K(\alpha, N) \int|x|^{\alpha-N} \frac{1-e^{-\operatorname{Re} \psi(x)}}{\operatorname{Re} \psi(x)} d x
$$

with $K(\alpha, N)$ finite if and only if $\alpha<N$ and with equality if $\psi$ is real valued. Thus $J(\alpha)<\infty$ if $\alpha<\beta^{\prime}$ and $\alpha<N$, and $J(\alpha)=\infty$ if $\alpha>\beta^{\prime}$ and $\psi$ real valued. The above calculation also shows that $J(\alpha)=\infty$ whenever $\psi$ is real valued and $\alpha \geqq N$, since in this case the constant $K(\alpha, N)=\infty$.

Theorem 5.3. (a) If $\alpha<\beta^{\prime}$ and $\alpha<N$, then

$$
t \varepsilon\left\{|X(t)|^{-\alpha}\right\} \rightarrow 0 \text { as } t \rightarrow 0 .
$$

(b) If $\alpha<\beta^{\prime \prime}$, then

$$
\varepsilon\left\{\exp \left[-t^{-1 / \alpha}|X(t)|\right]\right\} \rightarrow 0 \text { as } t \rightarrow 0 .
$$

Proof. (a) From (5.3) we have

$$
\mathcal{E}\left\{|X(t)|^{-\alpha}\right\} \leqq K \int_{0}^{\infty} u^{\alpha-1} d u \int e^{-\frac{1}{2}|y|^{2}} e^{-t \operatorname{Re} \psi(u y)} d y=K h(t) .
$$


Clearly $h(t)$ is non-increasing as $t$ increases and since $\alpha<\beta^{\prime}$ and $\alpha<N$ we have, exactly as in Theorem 5.2, that $\int_{0}^{1} h(t) d t<\infty$. Thus $\int_{0}^{t} h(\tau) d \tau \geqq h(t) t \rightarrow 0$ as $t \rightarrow 0$. This establishes (5.4).

Turning to (5.5) let

$$
h(t)=\varepsilon\left\{\exp \left[-t^{-1 / \alpha}|X(t)|\right]\right\}=\int e^{-t^{-1 / \alpha}|x|} F(t, d x) .
$$

Recalling the definition (3.2) of $f_{1}(x)$ we have

$$
\begin{aligned}
h(t) & =\iint e^{i(x, y)} t^{N / \alpha} f_{1}\left(t^{1 / \alpha} y\right) d y F(t, d x) \\
& =t^{N / \alpha} \int e^{-t \psi(y)} f_{1}\left(t^{1 / \alpha} y\right) d y \\
& \leqq t^{N / \alpha} \int_{|y| \leqq M}+\int_{|y|>M} e^{-t \operatorname{Re} \psi(y)} f_{1}\left(t^{1 / \alpha} y\right) d y \\
& =t^{N / \alpha}\left(I_{1}+I_{2}\right) .
\end{aligned}
$$

But $f_{1}\left(t^{1 / \alpha} y\right)$ is bounded, say by $M^{\prime}$, for $0 \leqq t \leqq 1$ and $|y| \leqq M$. Thus $t^{N / \alpha} I_{1} \rightarrow 0$ as $t \rightarrow 0$ for all finite $M$. Choose $\alpha^{\prime}$ such that $\alpha<\alpha^{\prime}<\beta^{\prime \prime}$; then if $M$ is large enough we have $\operatorname{Re} \psi(y) \geqq|y|^{\alpha^{\prime}}$ for $|y|>M$. Thus

$$
\begin{aligned}
t^{N / \alpha} I_{2} \leqq t^{N / \alpha} \int_{|y|>M} e^{-t|y| \alpha^{\prime}} f_{1}\left(t^{1 / \alpha} y\right) d y & \\
& =\int_{|y|>M} \exp \left[-t^{1-\alpha^{\prime} / \alpha}|y|^{\alpha^{\prime}}\right] f_{1}(y) d y \rightarrow 0
\end{aligned}
$$

as $t \rightarrow 0$. This proves (5.5).

Theorem 5.3 allows us to draw the following conclusion.

Corollary 5.1. If $\alpha<\beta^{\prime}$ and $\alpha<N$, then $t^{-1 / \alpha}|X(t)| \rightarrow \infty$ in probability as $t \rightarrow 0$. In any case if $\alpha<\beta^{\prime \prime}$, then $t^{-1 / \alpha}|X(t)| \rightarrow \infty$ in probability as $t \rightarrow 0$.

If $X_{1}$ and $X_{2}$ are independent symmetric stable processes in $R_{1}$ of indices $\alpha_{1}$ and $\alpha_{2}$ with $0<\alpha_{1}<\alpha_{2}<1$ then the process $Y(t)=\left(X_{1}(t), X_{2}(t)\right)$ in $R_{2}$ has stationary independent increments and exponent $\psi\left(x_{1}, x_{2}\right)=\left|x_{1}\right|^{\alpha_{1}}+\left|x_{2}\right|^{\alpha_{2}}$. For this process we clearly have $\beta^{\prime \prime}=\alpha_{1}$. On the other hand it is not difficult to see that $\beta^{\prime}=\alpha_{2}$ so that $\beta^{\prime \prime}<\beta^{\prime}$ is possible.

6. Subordinators. In this section we consider a particularly simple and important class of processes in $R_{1}$. A subordinator $\{T(t) ; t \geqq 0\}$ is a stochastic process in $R_{1}$ with stationary independent increments and with the additional property that $T(t)-T(s) \geqq 0$ with probability one whenever $t>s$. We assume, as usual, that $T(0)=0$ and that $T$ has the regularity properties described in section 2. If $\nu$ is the LÈvy measure of a real valued process $X$, then $X$ is a sub- 
ordinator if and only if the support of $\nu$ is contained in the half line $u \geqq 0$ and

$$
\int_{0}^{1} u \nu(d u)<\infty \text {. }
$$

Thus the index $\beta$ of a subordinator satisfies $\beta \leqq 1$.

In studying subordinators it is convenient to introduce the LAPLACE transform

$$
\varepsilon\left\{e^{-u T(t)}\right\}=e^{-t g(u)} \quad(u \geqq 0),
$$

where $g$ is necessarily of the form

$$
g(u)=\int_{0}^{\infty}\left(1-e^{-u r}\right) \nu(d r) .
$$

Clearly if we consider $u$ as a complex variable, then (6.3) defines $g$ in the right half plane $\operatorname{Re}(u) \geqq 0$. Also $\psi(u)=g(-i u)$ for all real $u$ where $\psi$ is the exponent of $T$. We will call $g$ the subordinator exponent of $T$. For a complete discussion of these and other properties of subordinators see BochNer [3], sections 4.1 and 4.3.

For a subordinator $T$ we will let $G(t, d u)$ denote the distribution (concentrated on $u \geqq 0$ ) of $T(t)$. In particular for each $\alpha$ with $0<\alpha<1$ there exists a subordinator, $T_{\alpha}$, such that $g(u)=u^{\alpha}$. Moreover $T_{\alpha}(t)$ has a continuous density. If we let $g_{\alpha}(u)$ be the density of $T_{\alpha}(1)$, then

$$
e^{-u^{\alpha}}=\int_{0}^{\infty} g_{\alpha}(r) e^{-u r} d r
$$

The process $T_{\alpha}$ is stable of index $\alpha$ and we will refer to it as the stable subordinator of index $\alpha$.

If $0<\alpha<1$ and $T$ is a subordinator we have

$$
\begin{aligned}
\int_{0}^{1} \mathcal{E}\left\{T(t)^{-\alpha}\right\} d t & =\int_{0}^{1} d t \int_{0}^{\infty} u^{-\alpha} G(t, d u) \\
& =\int_{0}^{1} d t \int_{0}^{\infty} \int_{0}^{\infty} \frac{1}{\Gamma(\alpha)} r^{\alpha-1} e^{-r u} d r G(t, d u) \\
& =\frac{1}{\Gamma(\alpha)} \int_{0}^{\infty} r^{\alpha-1} \frac{1-e^{-g(r)}}{g(r)} d r .
\end{aligned}
$$

Since $g \geqq 0$ and $g(r)$ is an increasing function of $r$ this last integral is finite if and only if

$$
\int_{1}^{\infty} \frac{r^{\alpha-1}}{g(r)} d r<\infty
$$

Thus we are led to introduce a special index $\sigma$ for subordinators, namely

$$
\sigma=\sup \left\{\alpha \leqq 1: \int_{1}^{\infty} \frac{r^{\alpha-1}}{g(r)} d r<\infty\right\} .
$$


It is immediate from Theorem 5.2 that

$$
\beta^{\prime \prime} \leqq \beta^{\prime} \leqq \sigma,
$$

and, from the definition, $\sigma \leqq 1$. The next theorem shows that $\sigma \leqq \beta$

Theorem 6.1. If $T$ is a subordinator then

(1) $\beta=\inf \left\{\alpha \geqq 0: \frac{g(u)}{u^{\alpha}} \rightarrow 0\right.$ as $\left.u \rightarrow \infty\right\}$

(2) $\sigma=\sup \left\{\alpha: \frac{g(u)}{u^{\alpha}} \rightarrow \infty \quad\right.$ as $\left.u \rightarrow \infty\right\}$

(3) $\sigma \geqq \sup \left\{\alpha \cdot u^{\alpha} \nu[u, \infty] \rightarrow \infty\right.$ as $\left.u \rightarrow 0\right\}$.

Proof. The proof of (1) is similar to the proof of Theorem 3.2 and will be omitted. Let $\gamma$ be the supremum on the right side of (2). If $\alpha<\sigma$ then

$$
\int_{1}^{\infty} \frac{u^{\alpha-1}}{g(u)} d u<\infty
$$

Now if one integrates by parts in (6.3) it is easy to see that $u^{-1} g(u)$ is a nonincreasing function of $u$. Therefore

$$
\int_{r}^{\infty} \frac{u^{\alpha-1}}{g(u)} d u=\int_{r}^{\infty} \frac{u}{g(u)} u^{\alpha-2} d u \geqq \frac{r}{g(r)} \frac{r^{\alpha-1}}{1-\alpha}=\frac{1}{1-\alpha} \frac{r^{\alpha}}{g(r)} .
$$

Since $\alpha<\sigma \leqq 1$ this implies $r^{\alpha} / g(r) \rightarrow 0$ as $r \rightarrow \infty$, and therefore $\alpha \leqq \gamma$. Hence $\sigma \leqq \gamma$.

Conversely if $\alpha<\gamma$ we choose $\alpha^{\prime}$ such that $\alpha<\alpha^{\prime}<\gamma$; then $u^{\alpha^{\prime}} / g(u) \rightarrow 0$ as $u \rightarrow \infty$. This implies that

$$
\int_{1}^{\infty} \frac{u^{\alpha-1}}{g(u)} d u<\infty
$$

and therefore $\alpha \leqq \sigma$. Hence $\sigma \geqq \gamma$, and this completes the proof of (2).

To prove (3) suppose $u^{\alpha} \nu[u, \infty] \rightarrow \infty$ as $u \rightarrow 0$; then

$$
\begin{aligned}
t^{\alpha} g\left(t^{-1}\right) & =t^{\alpha} \int_{0}^{\infty}\left(1-e^{-u / t}\right) \nu(d u) \\
& \geqq t^{\alpha} \int_{t}^{\infty}\left(1-e^{-u / t}\right) \nu(d u) \\
& \geqq\left(1-e^{-1}\right) t^{\alpha} \nu[t, \infty) .
\end{aligned}
$$

Thus $t^{\alpha} g\left(t^{-1}\right) \rightarrow \infty$ as $t \rightarrow 0$ or equivalently $r^{-\alpha} g(r) \rightarrow \infty$ as $r \rightarrow \infty$, that is $\alpha \leqq \sigma$. This establishes (3).

Note that (1) and (2) together yield

$$
\sigma \leqq \beta .
$$


For subordinators, Corollary 5.1 can be strengthened as follows.

Theorem 6.2. Let $T$ be a subordinator. Then

(1) if $\alpha<\sigma$ then $t^{-1 / \alpha} T(t) \rightarrow \infty$ with probability one as $t \rightarrow 0$;

(2) if $\alpha>\sigma$ then $\lim \inf t^{-1 / \alpha} T(t)=0$ with probability one.

Proof. To establish (1), choose $\alpha^{\prime}$ such that $\alpha<\alpha^{\prime}<\sigma$ and define $h(t)=$ $\mathcal{E}\left\{T(t)^{-\alpha^{\prime}}\right\}$. Then $\int_{0}^{1} h(t) d t<\infty$ since $\alpha^{\prime}<\sigma$. Now $P[T(t) \leqq \epsilon] \leqq \epsilon^{\alpha^{\prime}} \mathcal{E}\left\{T(t)^{-\alpha^{\prime}}\right\}$ for any $\epsilon>0$. Consider the events $A_{n}=\left\{T\left(2^{-n}\right)<2^{-n / \alpha^{\prime}}\right\}$. Then $P\left[A_{n}\right] \leqq$ $2^{-n} h\left(2^{-n}\right)$. Since $T(t)$ is non-decreasing with probability one, it follows that $h(t)$ is non-increasing. Therefore

$$
\sum_{n=1}^{\infty} P\left[A_{n}\right] \leqq \sum_{n=1}^{\infty} 2^{-n} h\left(2^{-n}\right) \leqq 2 \int_{0}^{1} h(t) d t<\infty,
$$

and thus the Borel-CANTELLI lemma implies that with probability one only finitely many of the $A_{n}$ occur. In other words there exists a subset $\Omega_{0}$ of $\Omega$ with $P\left[\Omega_{0}\right]=1$ such that $2^{n / \alpha^{\prime}} T\left(2^{-n}, \omega\right) \geqq 1$ for all but a finite number of $n$ if $\omega$ is in $\Omega_{0}$. Hence $2^{n / \alpha} T\left(2^{-n}, \omega\right) \rightarrow \infty$ as $n \rightarrow \infty$ if $\omega$ is in $\Omega_{0}$. However, if $2^{-(n+1)} \leqq$ $t<2^{-n}$ we have

$$
\begin{aligned}
t^{-1 / \alpha} T(t, \omega) & \geqq 2^{n / \alpha} T\left(2^{-(n+1)}, \omega\right) \\
& =2^{-1 / \alpha} 2^{(n+1) / \alpha} T\left(2^{-(n+1)}, \omega\right),
\end{aligned}
$$

and this implies $t^{-1 / \alpha} T(t, \omega) \rightarrow \infty$ as $t \rightarrow 0$ for all $\omega$ in $\Omega_{0}$. Thus (1) is proved.

We now turn our attention to (2). It follows from Theorem 6.1 that there exists a sequence $\left\{u_{n}\right\}$ with $u_{n} \rightarrow \infty$ and $u_{n}^{-\alpha} g\left(u_{n}\right) \rightarrow 0$ as $n \rightarrow \infty$. Then $t_{n}=$ $u_{n}^{-\alpha} \rightarrow 0$ as $n \rightarrow \infty$, and

$$
\mathcal{E}\left\{\exp \left[-t_{n}^{-1 / \alpha} T\left(t_{n}\right)\right]\right\}=\exp \left(-t_{n} g\left(t_{n}^{-1 / \alpha}\right)\right) \rightarrow 1 .
$$

Thus $t_{n}^{-1 / \alpha} T\left(t_{n}\right) \rightarrow 0$ in probability as $n \rightarrow \infty$ and a subsequence approaches 0 with probability 1 . So (2) is established.

Theorems 6.2, 3.1, and 3.3 give a complete description of the behavior of a subordinator near $t=0$ relative to simple HöLDER conditions in terms of the indices $\sigma$ and $\beta$.

7. A subordinator with $\sigma<\beta$. In this section we will show that $\beta^{\prime}<\beta$ is possible. In fact we will construct a whole class of subordinators for which $\sigma<\beta$. The construction depends on the following theorem.

Theorem 7.1. Let $T$ be a subordinator and suppose $\sigma=\beta<1$; then

$$
\sigma=\sup \left\{\alpha: u^{\alpha} \nu[u, \infty] \rightarrow \infty \quad \text { as } u \rightarrow 0\right\} .
$$

Proof. We have already seen in Theorem 6.1 that for any subordinator, $\sigma$ is not less than the supremum on the right of (7.1). Thus we need only prove the opposite inequality. If $\alpha<\sigma$ then we have from Theorem 6.1 that $u^{-\alpha} g(u) \rightarrow \infty$ 
as $u \rightarrow \infty$ or, equivalently, that $t^{\alpha} g\left(t^{-1}\right) \rightarrow \infty$ as $t \rightarrow 0$. Let $F(u)=\nu[u, \infty)$ so that

$$
g(t)=\int_{0}^{\infty}\left(e^{-t u}-1\right) d F(u)
$$

Since $\int_{0}^{1} u \nu(d u)<\infty$ we see that $u F(u) \rightarrow 0$ as $u \rightarrow 0$ (see the proof of Theorem 2.1). Using this last fact and integrating by parts we find that

$$
t^{-1} g(t)=\int_{0}^{\infty} e^{-u t} F(u) d u
$$

So for any $t>0$ and $\theta>0$ we have

$$
t^{\alpha} g\left(t^{-1}\right)=t^{\alpha-1} \int_{0}^{\theta} e^{-u / t} F(u) d u+t^{\alpha-1} \int_{\theta}^{\infty} e^{-u / t} F(u) d u=I_{1}+I_{2} .
$$

If $\alpha^{\prime}>\beta$ it follows from Theorem 2.1 that $u^{\alpha \prime} F(u) \rightarrow 0$ as $u \rightarrow 0$. Choose $\alpha^{\prime}$ such that $\beta<\alpha^{\prime}<1$ and let $\theta=\theta(t)=t(1-\alpha) /\left(1-\alpha^{\prime}\right)$; then $\theta(t) \rightarrow 0$ as $t \rightarrow 0$. But $F(u) \leqq u^{-\alpha^{\prime}}$ if $u$ is small and therefore we have

$$
I_{1} \leqq t^{\alpha-1} \int_{0}^{\theta} u^{-\alpha^{\prime}} d u=\frac{1}{1-\alpha^{\prime}} t^{\alpha-1} \theta^{1-\alpha^{\prime}}=\frac{1}{1-\alpha^{\prime}},
$$

provided $t$ is sufficiently small. However, $F$ is non-increasing and thus

$$
I_{2} \leqq t^{\alpha-1} F(\theta) \int_{\theta}^{\infty} e^{-u / t} d u \leqq t^{\alpha} F(\theta) .
$$

Now $t^{\alpha} g\left(t^{-1}\right) \rightarrow \infty$ as $t \rightarrow 0$ and so $t^{\alpha} F\left(t^{(1-\alpha) /\left(1-\alpha^{\prime}\right)}\right) \rightarrow \infty$ as $t \rightarrow 0$. This last result holds for all $\alpha<\sigma$ and $\alpha^{\prime}>\beta$ with $\alpha^{\prime}<1$. By hypothesis, $\sigma=\beta<1$ and therefore it follows easily that $t^{\alpha} F(t) \rightarrow \infty$ as $t \rightarrow 0$ for all $\alpha<\sigma$. This establishes Theorem 7.1.

Suppose $\nu$ is a measure on $(0, \infty)$ with $\int_{0}^{\infty} u(1+u)^{-1} \nu(d u)<\infty$ and such that for some $\alpha_{1}, \alpha_{2}$ with $0<\alpha_{1}<\alpha_{2}<1$ we have lim sup $u^{\alpha_{\mathrm{s}}} \nu[u, \infty) \geqq 1$ and $\lim \inf u^{\alpha_{1}} v[u, \infty) \leqq 1$ as $u \rightarrow 0$. It then follows from Theorems 2.1 and 7.1 that for the corresponding subordinator $T$, defined by

$$
\mathcal{E}\left\{e^{-u T(t)}\right\}=\exp \left[-t \int_{0}^{\infty}\left(1-e^{-u r}\right) \nu(d r)\right]
$$

we cannot have $\beta=\sigma$. The reader will have no difficulty constructing such a measure; in fact, $\nu$ may be chosen so that $F(u)=\nu[u, \infty)$ is completely monotonic. Thus the strict inequality $\sigma<\beta$ is indeed possible.

8. Dimension Theorems. In this section we will investigate the relationships between $\operatorname{dim} E$ and $\operatorname{dim} X(E)$. We will begin with a short summary of the relevant definitions and known facts concerning the notion of HAUSDORFFBesicovitch dimension. Before this, however, let us state the main result of this section. 
Theorem 8.1. Let $X$ be a process with stationary independent increments and $E$ a Borel subset of $[0,1]$ with $\operatorname{dim} E=\alpha$.

(1) If $\beta^{\prime} \leqq N$ then $P\left[\operatorname{dim} X(E) \geqq \alpha \beta^{\prime}\right]=1$. If $N=1$ and $\beta^{\prime}>1$, then at least $P\left[\operatorname{dim} X(E) \geqq \min \left(\alpha \beta^{\prime \prime}, 1\right)\right]=1$.

(2) If $\beta<1$, then $P[\operatorname{dim} X(E) \leqq \alpha \beta]=1$.

Let $\alpha$ be a positive real number and $E$ a subset of $R_{1}$ or $R_{N}$. For each $\epsilon>0$ set

$$
\Lambda_{\epsilon}^{\alpha}(E)=\inf \sum_{i=1}^{\infty}\left(\operatorname{diam} E_{i}\right)^{\alpha}
$$

where $\left\{E_{i} ; i \geqq 1\right\}$ is a cover of $E$ by subsets of $R_{N}$ all of diameter less than $\epsilon$, and the infimum is taken over all such covers. We would get the same number if we restricted the $E_{i}$ to be open sets or closed sets or, in the case of the real line, closed intervals. Let

$$
\Lambda^{\alpha}(E)=\lim _{\epsilon \rightarrow 0} \Lambda_{\epsilon}^{\alpha}(E) .
$$

Then $\Lambda^{\alpha}$ is called the HAUSDORFF $\alpha$-dimensional outer measure on $R_{N}$. It is a metric outer measure and so the Boneu sets are always measurable. We will need the following fact, which was proved by DAvies [4]: If $E$ is a Boreu set with $\Lambda^{\alpha}(E)=M \leqq \infty$ and if $0<h<M$, then there exists a closed set $F$ contained in $E$ such that $\Lambda^{\alpha}(F)=h$. It is also true that

$$
\sup \left\{\alpha: \Lambda^{\alpha}(E)=\infty\right\}=\inf \left\{\alpha: \Lambda^{\alpha}(E)=0\right\} .
$$

This common value is called the HaUsdorfF-Besicovitch dimension of $E$ and we will denote it by $\operatorname{dim} E$.

We need two more facts. First of all, a Bones subset $E$ of $R_{N}$ is said to have positive $\alpha$-capacity $\left(C_{\alpha}(E)>0\right)$ if there exists a probability measure, $m$, on $R_{N}$ with $m(E)=1$ such that

$$
\int_{E} \int_{E}|x-y|^{-\alpha} m(d x) m(d y)<\infty .
$$

Frostman ([5], p.. 86) has proved that if $E$ is closed and $\Lambda^{\alpha}(E)>0$, then $C_{\alpha^{\prime}}(E)>0$ for all $\alpha^{\prime}<\alpha$. In [1] and [2] the incorrect assertion was made that $\Lambda^{\alpha}(E)>0$ implies $C_{\alpha}(E)>0$ whenever $E$ is closed. Thus the proofs appearing on p. 269 (ii) of [1] and on p. 372 (i) of [2] must be slightly modified. Secondly we need the following theorem which is implicit in [8]. See also [1].

Theorem 8.2. Let $f$ be a measurable function from $[0,1]$ to $R_{N}$ and let $E$ be $a$ Borel subset of $[0,1]$. If there exists a probability measure $m$ on $[0,1]$ with $m(E)=1$ and such that

$$
\int_{E} \int_{E}|f(t)-f(s)|^{-\alpha} m(d t) m(d s)<\infty
$$

then $\Lambda^{\alpha}[f(E)]>0$. 
In order to prove the first statement of Theorem 8.1 we need several preliminary results which we will state and prove as lemmas.

Lemma 8.1. If $\alpha^{\prime}<\min \left(\beta^{\prime}, N\right)$ and if $\theta \leqq 1$ then $t^{\theta} \varepsilon\left\{|X(t)|^{-\theta \alpha^{\prime}}\right\} \rightarrow 0$ as $t \rightarrow 0$. Proof. Theorem 5.3(a) states that $t \mathcal{E}\left\{|X(t)|^{-\alpha^{\prime}}\right\} \rightarrow 0$ as $t \rightarrow 0$ and therefore $t^{\theta}\left(\varepsilon\left\{|X(t)|^{-\alpha^{\prime}}\right\}\right)^{\theta} \rightarrow 0$ as $t \rightarrow 0$. Since $\theta \leqq 1$ the function $f(u)=u^{\theta}$ is concave and thus for any positive random variable $Y$ we have $[\varepsilon\{Y\}]^{\theta} \geqq \varepsilon\left\{Y^{\theta}\right\}$. Hence

$$
t^{\theta}\left(\varepsilon\left\{|X(t)|^{-\alpha^{\prime}}\right\}\right)^{\theta} \geqq t^{\theta} \quad \mathcal{E}\left\{|X(t)|^{-\alpha^{\prime} \theta}\right\}
$$

and the lemma follows.

Lemma 8.2. If $\alpha^{\prime}<\beta^{\prime \prime}$ and $0<\gamma<N$, then there exist constants $M$ and $K$ (depending on $\alpha^{\prime}$ and $\gamma$ ) such that

$$
\varepsilon\left\{|X(t)|^{-\gamma}\right\} \leqq M+K t^{-\gamma / \alpha^{\prime}} .
$$

Proof. From equation (5.3) we easily obtain

$$
\begin{aligned}
\mathcal{E}\left\{|X(t)|^{-\gamma}\right\} & \leqq A \int_{0}^{\infty} u^{\gamma-1} d u \int e^{-\frac{1}{2}|y|^{2}} e^{-t \operatorname{Re} \psi(u y)} d y \\
& =A^{\prime} \int|y|^{\gamma-N} e^{-t \operatorname{Re} \psi(y)} d y .
\end{aligned}
$$

Moreover, $\operatorname{Re} \psi(y) \geqq|y|^{\alpha^{\prime}}$ for $|y|$ large enough. Thus there exists $R>0$ such that

$$
\begin{aligned}
\mathcal{E}\left\{|X(t)|^{-\gamma}\right\} & \leqq A^{\prime} \int_{|y| \leqq R}|y|^{\gamma-N} d y+A^{\prime} \int_{|y|>R}|y|^{\gamma-N} e^{-t|y| \alpha^{\prime}} d y \\
& \leqq M+A^{\prime \prime} t^{-\gamma / \alpha^{\prime}} \int_{0}^{\infty} u^{\gamma / \alpha^{\prime}-1} e^{-u} d u \\
& =M+K t^{-\gamma / \alpha^{\prime}} .
\end{aligned}
$$

We are now ready to prove Theorem 8.1 (1). First of all consider the case $\beta^{\prime} \leqq N$. If $\gamma<\alpha \beta^{\prime}$ then we can choose $\alpha^{\prime}$ and $\alpha^{\prime \prime}$ such that

$$
\gamma / \alpha<\alpha^{\prime \prime}<\alpha^{\prime}<\beta^{\prime} \leqq N .
$$

This implies $\gamma / \alpha^{\prime \prime}<\alpha$ and thus $\Lambda^{\gamma / \alpha^{\prime \prime}}(E)=\infty$. By Davies' theorem there exists a closed subset $F$ of $E$ such that $\Lambda^{\gamma / \alpha^{\prime}}(F)>0$. Now Frostman's theorem implies that $C_{\gamma / \alpha^{\prime}}(F)>0$; that is, there exists a probability measure, $m$, on $[0,1]$ with $m(F)=1$ such that

$$
\int_{F} \int_{F}|t-s|^{-\gamma / \alpha^{\prime}} m(d t) m(d s)<\infty .
$$

Applying Lemma 8.1 with $\theta=\gamma / \alpha^{\prime}<\alpha<1$ we see that

$$
\varepsilon\left\{|X(t)-X(s)|^{-\gamma}\right\}=\varepsilon\{|X(|t-s|)|\}^{-\left(\gamma / \alpha^{\prime}\right) \alpha^{\prime}} \leqq|t-s|^{-\gamma / \alpha^{\prime}}
$$


provided $|t-s|$ is small enough. But $\mathcal{E}\left\{|X(|t-s|)|^{-\gamma}\right\}$ is bounded if $|t-s| \geqq$ $\delta>0$ and thus applying the FuBINI theorem we find that for almost all $\omega$

$$
\int_{F} \int_{F}|X(t, \omega)-X(s, \omega)|^{-\gamma} m(d t) m(d s)<\infty .
$$

Now from Theorem 8.2 we obtain $\Lambda^{\gamma}[X(E)] \geqq \Lambda^{\gamma}[X(F)]>0$ with probability one. Therefore $P[\operatorname{dim} X(E) \geqq \gamma]=1$ and since $\gamma<\alpha \beta^{\prime}$ was arbitrary the proof is complete in this case.

In the remaining case $N=1$ and $\beta^{\prime}>1$ one argues similarly using Lemma 8.2 in place of Lemma 8.1. We omit the details.

In order to prove Theorem 8.1 (2) we will need several preliminary facts which are of independent interest. Suppose $\beta<1$ and choose $\alpha$ such that $\beta<\alpha \leqq 1$; then the quantity $V_{\alpha}(t)=V_{\alpha}[X(\cdot, \omega) ; 0, t]$ is finite with probability one according to Theorem 4.2. (Actually we only proved this for $t=1$ but it follows easily, since $\alpha \leqq 1$, that there exists a set $\Omega_{0}$ with $P\left[\Omega_{0}\right]=1$ such that $V_{\alpha}(t)<\infty$ for all $t<\infty$ and $\omega$ in $\Omega_{0}$.) Also using the fact that $\alpha \leqq 1$ again (namely, the inequality $|a+b|^{\alpha} \leqq|a|^{\alpha}+|b|^{\alpha}$ ) it is easy to verify that $V_{\alpha}(t+s)-V_{\alpha}(s)=V_{\alpha}[X ; s, t+s]$, and therefore the process $V_{\alpha}=\left\{V_{\alpha}(t)\right.$; $t \geqq 0\}$ has stationary independent increments. Since $V_{\alpha}(t)$ is clearly nondecreasing we see that $V_{\alpha}$ is a subordinator. Using an obvious notation we have the following result.

Theorem 8.3. If $X$ is a process with $\beta(X)<\alpha \leqq 1$ then $\beta\left(V_{\alpha}\right) \leqq \beta(X) / \alpha$.

Proof. Choose $\alpha^{\prime}$ such that $\beta(X) / \alpha<\alpha^{\prime} \leqq 1$, and consider expressions of the form

$$
\sum_{i=1}^{n}\left|V_{\alpha}\left(t_{i}\right)-V_{\alpha}\left(t_{i-1}\right)\right|^{\alpha^{\prime}}
$$

where $0=t_{0}<\cdots<t_{n}=1$. Each summand may be approximated arbitrarily closely by an expression of the form

$$
\left(\sum_{j=1}^{m_{i}}\left|X\left(t_{i, j}\right)-X\left(t_{i, i-1}\right)\right|^{\alpha}\right)^{\alpha^{\prime}} \leqq \sum_{j=1}^{m_{i}}\left|X\left(t_{i, j}\right)-X\left(t_{i, j-1}\right)\right|^{\alpha \alpha^{\prime}}
$$

where $t_{i-1}=t_{i, 0}<t_{i, 1}<\cdots<t_{i, m i}=t_{i}$. Thus $V_{\alpha^{\prime}}\left(V_{\alpha}\right) \leqq V_{\alpha \alpha^{\prime}}(X)<\infty$ because $\beta(X)<\alpha \alpha^{\prime} \leqq 1$. Now Theorem 4.2 implies $\beta\left(V_{\alpha}\right) \leqq \alpha^{\prime}$, and since $\alpha^{\prime}$ with $\beta(X) / \alpha<\alpha^{\prime} \leqq 1$ was arbitrary, the proof of Theorem 8.3 is complete.

Actually with a more detailed analysis using the ITo representation of $X$ [9], appropriately generalized to $R_{N}$, one can show that $\beta\left(V_{\alpha}\right)=\beta(X) / \alpha$. We do not need this result and so we omit the proof. However, if $X$ is stable with index $\beta<1$, then it is quite easy to see that $V_{\alpha}$ is again stable with index $\beta / \alpha$ This gives an interesting transformation between the sample functions of the general stable processes and those of the one-sided stable processes in $R_{\mathbf{1}}$, that is, the stable subordinators.

We need two more facts, which we state as lemmas. The functions $g_{\alpha}(0<\alpha<1)$ are defined in equation (6.4). 
Lemma 8.3. If $g$ is a subordinator exponent of index $\beta$ and if $\theta>\beta, \gamma \leqq 1$, and $\theta \gamma<1$, then $\int_{0}^{\infty}(g(u))^{\gamma} g_{\theta \gamma}(u) d u<\infty$.

Proof. We first note that if $g$ is any subordinator exponent of index $\beta$ and if $\beta<\alpha<1$ then

$$
\begin{aligned}
\int_{0}^{\infty} g(u) g_{\alpha}(u) d u & =\int_{0}^{\infty} \int_{0}^{\infty}\left(1-e^{-r u}\right) \nu(d r) g_{\alpha}(u) d u \\
& =\int_{0}^{\infty}\left(1-e^{-r \alpha}\right) \nu(d r) .
\end{aligned}
$$

This last integral is finite if and only if $\int_{0}^{1} r^{\alpha} \nu(d r)<\infty$ and this is the case since $\alpha>\beta$. This proves Lemma 8.3 if $\gamma=1$. Thus in the remainder of the proof we can assume $\gamma<1$. Now Bochner ([3], Section 4.3) has shown that if $h(u)$ and $g(u)$ are subordinator exponents, then so is $h[g(u)]$. Since $h(u)=u^{\gamma}$ is a subordinator exponent if $0<\gamma<1$ (corresponding to the stable subordinator of index $\gamma$ ), it follows that $[g(u)]^{\gamma}$ is a subordinator exponent. Moreover, it is an easy consequence of Theorem 6.1 (1) that the index, $\widetilde{\beta}$, of $(g(u))^{\gamma}$ satisfies $\tilde{\beta} \leqq \beta \gamma$. Since $\beta \gamma<\theta \gamma$ the proof of Lemma 8.3 is complete.

Lemma 8.4. Let $T$ be a subordinator with index $\beta<1$. Suppose $\beta<\gamma<1$ and $0<\theta \leqq 1$ and define

$$
A(t)=\int_{0}^{\infty} e^{-u^{\theta \gamma}} G(t, d u)
$$

Then $t^{-\theta}[1-A(t)]$ remains bounded as $t \rightarrow 0$.

Proof. Since $\theta \gamma<1$ we can write

$$
I(t)=t^{-\theta}[1-A(t)]=t^{-\theta} \int_{0}^{\infty}\left[1-e^{-t g(u)}\right] g_{\theta \gamma}(u) d u \text {. }
$$

For fixed $t>0$ define $B=\{u: \operatorname{tg}(u)>1\}$ and let $B^{\prime}$ be the complement of $B$ in $[0, \infty)$; then

$$
\begin{aligned}
I(t) & \leqq t^{-\theta} \int_{B^{\prime}}\left[1-\exp \left(-(\operatorname{tg}(u))^{\theta}\right)\right] g_{\theta \gamma}(u) d u+t^{-\theta} \int_{B} g_{\theta \gamma}(u) d u \\
& \leqq 2 \int_{0}^{\infty}(g(u))^{\theta} g_{\theta \gamma}(u) d u .
\end{aligned}
$$

This last integral is finite by Lemma 8.3 , so the proof is complete.

We are now in position to prove Theorem 8.1(2) at least in the case $\alpha=$ $\operatorname{dim} E<1$.

Choose $\gamma$ so that $\beta(X)<\gamma \leqq 1$ and let $V_{\gamma}(t)=V_{\gamma}(X(\cdot, \omega) ; 0, t)$; then $V_{\gamma}$ is a subordinator with $\beta\left(V_{\gamma}\right) \leqq \beta(X) / \gamma<1$. If $\beta\left(V_{\gamma}\right)<\eta<1$, then for any $\theta \leqq 1$ set

$$
\left.\widetilde{A}(t)=\int_{0}^{\infty} \exp \left(-u^{\eta \theta}\right) G(t, d u)=\varepsilon\left\{\exp \left[-V_{\gamma}(t)\right)^{\eta \theta}\right]\right\}
$$


where $G(t, \cdot)$ is the distribution of the subordinator $V_{\gamma}(t)$. According to Lemma 8.4 we have that $t^{-\theta}[1-\widetilde{A}(t)]$ remains bounded as $t \rightarrow 0$.

If $d(t)=\operatorname{diam} X([0, t])$, then $(d(t))^{\gamma} \leqq V_{\gamma}(t)$ for any $t>0$. Thus if we let

$$
A(t)=e^{-B(t)}=\mathcal{E}\left\{\exp \left[-d(t)^{\gamma \eta \theta}\right]\right\}
$$

we have $1 \geqq A(t) \geqq \widetilde{A}(t)$ and so $t^{-\theta}[1-A(t)]$ remains bounded as $t \rightarrow 0$. It follows that there exists a finite constant $K$ such that

$$
B(t) \leqq K t^{\theta}
$$

for $0 \leqq t \leqq 1$.

Given $E$ contained in [0, 1] with $\operatorname{dim} E=\alpha<1$ pick $\alpha^{\prime}$ such that $\alpha<\alpha^{\prime}<1$ and for each positive integer $n$ let $\left\{E_{n i} ; i \geqq 1\right\}$ be a cover of $E$ by non-overlapping (i.e. with only end points in common) intervals such that

$$
\sum_{i}\left(\operatorname{diam} E_{n i}\right)^{\alpha^{\prime}}<\frac{1}{n} .
$$

This is possible since $\Lambda^{\alpha^{\prime}}(E)=0$. Clearly $\left\{X\left(E_{n i}\right) ; i \geqq 1\right\}$ is a cover of $X(E)$, and if we apply the foregoing estimates with $\theta=\alpha^{\prime}$ we obtain

$$
\begin{aligned}
\mathcal{E}\left\{\operatorname { e x p } \left[-\sum_{i}\right.\right. & \left.\left.\left(\operatorname{diam} X\left(E_{n i}\right)\right)^{\gamma \eta \alpha^{\prime}}\right]\right\}=\prod_{i} A\left(\operatorname{diam} E_{n i}\right) \\
& =\exp \left[-\sum_{i} B\left(\operatorname{diam} E_{n i}\right)\right] \\
& \geqq \exp \left[-K \sum_{i}\left(\operatorname{diam} E_{n i}\right)^{\alpha^{\prime}}\right] \geqq e^{-K / n}
\end{aligned}
$$

Here we have used the facts that for each $n$ the random variables diam $X\left(E_{n i}\right)$ are mutually independent for $i \geqq 1$ and that their distribution depends only on diam $E_{n i}$. Therefore $\sum_{i}$ [diam $\left.X\left(E_{n i}\right)\right]^{\gamma \eta \alpha^{\prime}}$ approaches zero in probability as $n \rightarrow \infty$ and thus a subsequence does so with probability one. This implies $P\left[\operatorname{dim} X(E) \leqq \gamma \eta \alpha^{\prime}\right]=1$. But $\gamma \eta\langle\gamma, \gamma\rangle \beta(X)$ was arbitrary and so was $\alpha^{\prime}>\alpha$, and therefore we have $P[\operatorname{dim} X(E) \leqq \alpha \beta(X)]=1$, which completes the proof of Theorem 8.1(2) if $\alpha<1$.

The case $\alpha=1$ remains. However, in this case the validity of our assertion is an immediate consequence of the following fact.

Theorem 8.4. If $f:[0, \infty) \rightarrow R_{N}$ has the following properties:

(a) $f$ is everywhere right continuous,

(b) f has left-hand limits everywhere,

(c) $f$ is bounded on bounded intervals, then $\Lambda^{\alpha} f([0,1]) \leqq 2^{\alpha} V_{\alpha}(f ; 0,1)$ for every $\alpha>0$.

Proof. Given $\epsilon>0$ define the sequence $\left\{t_{n}\right\}$ inductively as follows: $t_{0}=0$ and, for $n \geqq 1$,

$$
t_{n}=\inf \left\{t \geqq t_{n-1}:\left|f(t)-f\left(t_{n-1}\right)\right|>\epsilon / 2\right\} .
$$


By the right continuity of $f$ we have $t_{0}<t_{1}<t_{2}<\cdots$, and by the left-hand limits requirement, $t_{n}>1$ for large enough $n$. Let $n_{0}=\min \left\{n: t_{n}>1\right\}$. If $A_{i}=f\left(\left[t_{i-1}, t_{i}\right)\right)$, then $A_{1}, \cdots, A_{n_{0}}$ is a cover of $f([0,1])$ with $\operatorname{diam} A_{i} \leqq \epsilon$. The right continuity of $f$ implies that $\left|f\left(t_{i}\right)-f\left(t_{i-1}\right)\right| \geqq \epsilon / 2$ and therefore $\operatorname{diam} A_{i} \leqq 2\left|f\left(t_{i}\right)-f\left(t_{i-1}\right)\right|$. Thus

$$
\begin{aligned}
\Lambda_{\epsilon}^{\alpha} f([0,1]) & \leqq 2^{\alpha} \sum_{i=1}^{n_{0}-1}\left|f\left(t_{i}\right)-f\left(t_{i-1}\right)\right|^{\alpha}+\epsilon^{\alpha} \\
& \leqq 2^{\alpha} V_{\alpha}(f ; 0,1)+\epsilon^{\alpha},
\end{aligned}
$$

and so $\Lambda^{\alpha} f([0,1]) \leqq 2^{\alpha} V_{\alpha}(f ; 0,1)$.

Theorem 8.1(2) in the case $\alpha=1$ now follows from Theorems 4.2 and 8.4 since almost all sample functions of the process $\{X(t) ; t \geqq 0\}$ satisfy the regularity conditions of Theorem 8.4. Thus the proof of Theorem 8.1 is finally complete.

For subordinators one can strengthen Theorem 8.1 slightly (we do not require $\beta<1$; of course, $\beta \leqq 1$ for a subordinator) to obtain the following theorem.

Theorem 8.5. If $T$ is a subordinator and $E$ is a subset of $[0,1]$ with $\operatorname{dim} E=\alpha$, then $P[\alpha \sigma \leqq \operatorname{dim} T(E) \leqq \alpha \beta]=1$

Finally let us remark that Theorem 8.1(2) should be true without the restriction $\beta<1$, but we have been unable to establish it in this generality In [2] we were able to prove it for the general stable processes without the restriction $\beta<1$

9. Some General Remarks. In the previous section we obtained a number of estimates relating $\operatorname{dim} X(E)$ to $\operatorname{dim} E$. Now it is reasonable to conjecture that given a BorEL subset $E$ of $[0,1]$ there exists a constant $\lambda(X, E)$ such that

$$
P[\operatorname{dim} X(E)=\lambda(X, E)]=1 \text {. }
$$

Moreover if we let $\lambda(X)=\lambda(X,[0,1])$, then it may be true that

$$
\lambda(X, E)=\lambda(X) \operatorname{dim} E .
$$

In particular Theorem 8.1 implies that if $\beta^{\prime \prime}=\beta<1$, then $\lambda(X)=\beta$ and (9.2) holds. Also the results of [2] state that if $X$ is a stable process of index $\alpha$ then $\lambda(X)=\alpha$ and (9.2) holds. In the general case we have only been able to prove the existence of $\lambda(X, E)$ if $E$ is closed, and have made no progress towards proving (9.2).

We show the existence of $\lambda(X, E)$ in two steps: (1) we show that $\Delta=$ $\left\{\omega: \Lambda^{\alpha}[X(E, \omega)]>0\right\}$ is measurable: (2) assuming $\Delta$ measurable we show that $P[\Delta]$ is zero or one. As might be expected the first step causes the most trouble. In any case we will prove (2) for general $E$, but will only be able to establish (1) for closed $E$. Statements (1) and (2) are a bit imprecise, but this will be remedied as we proceed. 
In what follows if $Q$ is any collection of subsets of a set $\Omega$ then $B(Q)$ denotes the smallest $\sigma$-algebra containing $Q$.

Lemma 9.1. Let $(\Omega, F, P)$ be a probability space and suppose that $\left(\digamma_{n}, \mathcal{G}_{n}\right)$ is a sequence of pairs of $\sigma$-subalgebras of $\mathcal{F}$ such that for each $n, \mathcal{F}_{n}$ and $\mathcal{G}_{n}$ are independent. Suppose further that $B\left(\cup_{n} F_{n}\right)=\mathcal{F}$. If $B$ is in $\mathcal{G}_{n}$ for every $n$ then $P[B]=0$ or 1 .

Proof. The usual zero-one argument leads to the equation $P[B \cap B]=$ $P[B] \cdot P[B]$.

Let $x$ denote the set of all functions $x(\cdot):[0,1] \rightarrow R_{N}$, which have left-hand limits everywhere, and are bounded and everywhere right-continuous. And let $B$ denote the $\sigma$-algebra of subsets of $X$ generated by all sets of the form $\{x(\cdot): x(t)$ in $A\}$ where $t$ ranges over $[0,1]$, and $A$ over the BonEL sets of $R_{N} . X$ will denote a process of the type we have been considering; by deleting from $\Omega$ a single $P$-null set we may assume that all sample functions of $X$ are as regular as the elements of $x$.

Theorem 9.1. Let $E$ be a subset of $[0,1]$ and let $\widetilde{E}=\left\{x(\cdot): \Lambda^{\alpha}(x(E))>0\right\}$ be in B. If $\Delta=\{\omega: X(\cdot, \omega)$ in $\widetilde{E}\}\left(=\left\{\omega \mid \Lambda^{\alpha}(X(E, \omega))>0\right\}\right)$ then $P[\Delta]=0$ or 1 .

Proof. In the present proof we will need to make use of the ITo representation of the process $X$; see [9]. This can be described as follows. Let $\Pi=[0, \infty) \times R_{N}$ and denote the points of $\prod$ by $(t, x)$ where $t \geqq 0$ and $x$ in $R_{N}$. Let $\beta_{0}$ be the class of all Bores sets in $\prod$ which are bounded and at a positive distance from the " $x$-axis". Clearly $\beta_{0}$ is a ring of sets. For $\omega$ in $\Omega$ and $B$ in $\beta_{0}$ define $N(\omega, B)$ to be the number of points $(t, x)$ in $B$ such that $X(t, \omega)-X\left(t^{-}, \omega\right)=x$. Now for fixed $B$ in $\beta_{0}$ the random variable $N(\cdot, B)$ has a PoIsson distribution with mean value $\iint_{B} d t \nu(d x)$ where $\nu$ is the Lìvy measure of $X$. Also $N(\omega, \cdot)$ is a measure on $\beta_{0}$ for fixed $\omega$ in $\Omega$, which takes on only non-negative integral values. Moreover, if $B_{1}, \cdots, B_{n}$ is any finite collection of disjoint sets in $B_{0}$, then the random variables $N\left(\cdot, B_{1}\right), \cdots, N\left(\cdot, B_{n}\right)$ are mutually independent. Let $Q_{0}$ be the ring of all bounded Bones sets in $R_{N}$ at a positive distance from the origin and define $N(t, \omega, A)=N(\omega,[0, t] \times A)$. Then $\mathcal{\varepsilon}\{N(t, \omega, A)\}=$ $t \nu(A)$ and $N(t, \omega, A)$ is the number of "jumps" $X(\tau, \omega)-X\left(\tau^{-}, \omega\right)$ in $A$ for $0 \leqq \tau \leqq t$. Finally we have the following representation of the process $\mathrm{X}$ :

$$
X(t)=a t+\lim _{n \rightarrow \infty} \int_{1 / n<|x|<n}\left[x N(t, \omega, d x)-\frac{t x}{1+|x|^{2}} \nu(d x)\right]
$$

where $a$ is in $R_{N}$ and the limit exists with probability one uniformly on bounded $t$-intervals. (The integrals in (9.3) are, of course, vector integrals in $R_{N}$.) Actually ITo proved this result for processes in $R_{1}$, but the proof carries over to $R_{N}$ without difficulty and we omit the details. Finally by deleting a single $P$-null set we may assume that the limit in (9.3) holds for all $\omega$ and that $N(t, \omega, A)$ is finite for all $\omega$, all $t \geqq 0$, and all $A$ in $a_{0}$.

We now return to the proof of Theorem 9.1. For each integer $k>0$ let $B_{k}=$ $\left\{x:|x|<k^{-1}\right\}$ and $B_{k}^{\prime}=\left\{x:|x| \geqq k^{-1}\right\}$. Take $\mathcal{F}$ in Lemma 9.1 to be $\sigma$-algebra of 
the process $X$ and define $\sigma$-subalgebras of $\mathcal{F}$ as follows: $\mathscr{F}_{k}$ is the $\sigma$-algebra determined by the random variables $N(t, \cdot, A)$ with $t \geqq 0, A$ in ${Q_{0}}_{0}$, and $A \subset B_{k}^{\prime}$; $\mathcal{G}_{k}$ is the $\sigma$-algebra determined by $N(t, \cdot, A)$ with $t \geqq 0, A$ in $Q_{0}$, and $A \subset B_{k}$. Clearly $\mathscr{F}=\mathbb{B}\left(\cup \mathfrak{F}_{k}\right)$ and the pair $\left(\mathfrak{F}_{k}, \mathcal{G}_{k}\right)$ are independent. We next show that $\Delta$ is in $\mathcal{G}_{k}$ for all $k$. To this end we define a process $X_{k}$ by

$$
X_{k}(t)=a t+\lim _{n \rightarrow \infty} \int_{1 / n<|x|<1 / k}\left[x N(t, \omega, d x)-\frac{t x}{1+|x|^{2}} \nu(d x)\right] .
$$

Loosely speaking, $X_{k}$ is the process one gets from $X$ by throwing out all jumps of magnitude greater than or equal to $k^{-1}$. More precisely, there exists a finite number $n_{k}(\omega)$ such that

$$
X(t, \omega)-X_{k}(t, \omega)=\sum_{j=1}^{n_{k}} u\left(t-t_{i}\right) x_{j}
$$

for all $\omega$ where $u(t)$ is 0 for $t<0$ and 1 for $t \geqq 0$. The values $t_{j}$ and $x_{i}$, of course, depend on $\omega$. Thus, as is easy to see, for all $\omega$ we have $X(\cdot, \omega)$ in $E$ if and only if $X_{k}(\cdot, \omega)$ is in $E$. Clearly $X_{k}$ is $\mathcal{G}_{k}$ measurable and so $\Delta$ is in $\mathcal{G}_{k}$ for all $k$. Thus by Lemma $9.1, P[\Delta]$ is either zero or one.

We now consider the measurability hypothesis of Theorem 9.1 .

Theorem 9.2. If $E$ is a subset of $[0,1]$ then the function $f: x \rightarrow[0, \infty]$ defined by $f(x(\cdot))=\Lambda^{\alpha}(\overline{x(\bar{E})})$ is B-measurable.

The notation $\bar{A}$ denotes the closure of $A$.

Proof. In the proof we assume, for simplicity, that $N=1$. Only minor changes are necessary to carry out the argument for general $N$. It is easy to see that if $A$ is a compact subset of $R_{1}$, then

$$
\Lambda_{\epsilon}^{\alpha}(A)=\inf \sum_{i=1}^{n}\left(\operatorname{diam} E_{i}\right)^{\alpha}
$$

where the infimum is taken over all finite covers $\left\{E_{i} ; i \leqq n\right\}$ of $A$ with each $E_{i}$ being an open interval with rational end points and length not exceeding $\epsilon$.

Let $B$ be any open bounded subset of $R_{1}$ and let $x_{k}$ be a countable dense subset of $B^{\prime}$ (the complement of $B$ ); then

$$
\{x(\cdot): B \text { covers } \overline{x(E)}\}=\bigcup_{n=1}^{\infty}\left\{x(\cdot): d\left[\overline{x(E)}, B^{\prime}\right] \geqq \frac{1}{n}\right\}
$$

where $d$ is the usual distance between subsets of $R_{1}$. Also

$$
\left\{x(\cdot): d\left[\overline{x(E)}, B^{\prime}\right]<\frac{1}{n}\right\}=\bigcup_{r} \bigcup_{k=1}^{\infty}\left\{x(\cdot):\left|x(r)-x_{k}\right|<\frac{1}{n}\right\}
$$

where $r$ ranges over a countable subset of $E$ having the property that any $t$ in 
$E$ is itself an $r$ or is a limit from the right of a sequence of $r$ 's. Each set on the right side of (9.6) is in $B$ and therefore so is the set on the left side of (9.5).

Given $\epsilon>0$ let $\left\{B_{n} ; n \geqq 1\right\}$ be an enumeration of the subsets of $R_{1}$ of the form $E_{1} \cup \ldots \cup E_{k}$ where each $E_{i}$ is an open interval with rational end points and length not exceeding $\epsilon$. Note that $B_{i}$ and $B_{i}$ might refer to different decompositions of the same set. If $B_{n}=E_{1} \cup \cdots \cup E_{k}$ set

$$
l_{\alpha}\left(B_{n}\right)=\sum_{i=1}^{k}\left(\operatorname{diam} E_{i}\right)^{\alpha} .
$$

Since $\overline{x(E)}$ is compact we have $\Lambda_{\epsilon}^{\alpha}(\overline{x(E)})<u$ if and only if $l_{\alpha}\left(B_{n}\right)<u$ for some $B_{n}$ which covers $\overline{x(E)}$. Given $u>0$ let $\left\{B_{n, u} ; n \geqq 1\right\}$ consist of those $B_{n}$ for which $l_{\alpha}\left(B_{n}\right)<u$. Then

$$
\left\{x(\cdot): \Lambda_{\epsilon}^{\alpha}(x(E))<u\right\}=\bigcup_{n=1}^{\infty}\left\{x(\cdot): B_{n, u} \text { covers } \overline{x(E)}\right\} .
$$

We have already shown that each set in the union on the right is in $B$ and therefore $\Lambda_{\epsilon}^{\alpha}(\overline{x(E)})$ is a $B$ measurable function. Now letting $\epsilon \rightarrow 0$ through a countable sequence yields the measurability of $f$.

Corollary 9.1. If $E$ is closed, then $\Lambda^{\alpha}(x(E))$ is \& measurable.

Proof. In this case $\overline{x(E)}=x(E)$ together with all points $y$ that can be obtained as $y=\lim x\left(s_{n}\right)$ where $\left\{s_{n}\right\}$ is any non-decreasing sequence of points in $E$. This limit always exists and, of course, $\lim s_{n}=t$ with $t$ in $E$. But $\lim x\left(s_{n}\right)=x(t)$ for all but countably many $t$, since $x(\cdot)$ is right continuous and therefore has only countably many discontinuities. Thus $\overline{x(E)}$ differs from $x(E)$ by a countable set and since any countable set has zero $\Lambda^{\alpha}$-measure the corollary is established. In particular $\widetilde{E}$ is in $B$ if $E$ is closed.

Corollary 9.1 and Theorem 9.1 taken together imply the existence of the constant $\lambda(X, E)$ for any closed set $E$. The problem is still open for more general E.

We would particularly like to thank H. P. McKean for his suggestions in connection with this section.

\section{ReFERENCES}

[1] R. M. Blumenthal \& R. K. Getoon, Some theorems on stable processes, Trans. Amer. Math. Soc., 95 (1960) 263-273.

[2] $\longrightarrow$ A dimension theorem for sample functions of stable processes, $\mathrm{Ill}$. Journ. of Math., 4 (1960) 370-375.

[3] S. Bochner, Harmonic Analysis and the Theory of Probability, Berkeley and Los Angeles, 1955.

[4] R. O. Davies, Subsets of finite measure in analytic sets, Indag. Math., 14 (1952) 488-489.

[5] O. Frostman, Potential d'equilibre et capacité des ensembles avec quelques applications à la théorie des fonctions, Meddelanden fran Lunds Univ. Math. Sem., 3 (1935). 
[6] G. A. Hunt, Some theorems concerning Brownian motion, Trans. Amer. Math. Soc., 81 (1956) 294-319.

[7] A. Khintchine, Sur la croissance locale des processus stochastique homogènes à acroissements indépendants, Izvestia Akad. Nauk, SSSR, Ser. Math., (1939) 487-508; Math. Rev., 1 (1940) 344.

[8] H. P. McKean Jr., Sample functions of stable processes, Ann. Math., 61 (1955) 564-579.

[9] K. Iто, On stochastic processes, Jap. Journ. of Math., 18 (1942) 261-301.

[10] K. Takano, On some limit theorems of probability distributions, Annals of the Institute of Statistical Mathematics, 6 (1954) 37-113.

University of Washington

Seattle, Washington 